02

\title{
Фононный спектр и упругие свойства $\mathrm{Y}_{2} \mathrm{Sn}_{2} \mathrm{O}_{7}$
}

\author{
(C) В.А. Чернышев ${ }^{1}$, К.И. Глухов ${ }^{1}$, П.А. Агзамова ${ }^{1,2}$ \\ ${ }^{1}$ Уральский фредеральный университет им. Б.Н. Ельцина, \\ 620075 Екатеринбург, Россия \\ ${ }^{2}$ Институт фризики металлов имени М.Н. Михеева Уральского отделения РАН, \\ 620108 Екатеринбург, Россия \\ e-mail: vladimir.chernyshev@urfu.ru
}

Поступила в редакцию 24.03.2021 г.

В окончательной редакции 19.04.2021 r.

Принята к публикации 27.04.2021 г.

В рамках единого ab initio подхода проведен расчет фононного спектра станната иттрия $\mathrm{Y}_{2} \mathrm{Sn}_{2} \mathrm{O}_{7}$, определены частоты и типы ИК и КРС-мод. Из анализа векторов смещений, полученных при ab initio расчете, определена степень участия ионов в фононных модах. Рассчитаны упругие постоянные и твердость $\mathrm{Y}_{2} \mathrm{Sn}_{2} \mathrm{O}_{7}$. Рассчитано расстояние „примесный ион-лиганд“ в примесных центрах $\mathrm{Y}_{2} \mathrm{Sn}_{2} \mathrm{O}_{7}: \mathrm{Yb}^{3+}, \mathrm{Y}_{2} \mathrm{Sn}_{2} \mathrm{O}_{7}: \mathrm{Eu}^{3+}$, $\mathrm{Lu}_{2} \mathrm{Sn}_{2} \mathrm{O}_{7}: \mathrm{Yb}^{3+}, \mathrm{Nd}_{2} \mathrm{Sn}_{2} \mathrm{O}_{7}: \mathrm{Gd}^{3+}, \mathrm{Nd}_{2} \mathrm{Sn}_{2} \mathrm{O}_{7}: \mathrm{Tb}^{3+}$.

Ключевые слова: редкоземельные станнаты, фононы, упругие постоянные, редкоземельные примесные центры, гибридные функционалы.

DOI: $10.21883 / O S .2021 .08 .51198 .2056-21$

\section{Введение}

Кристаллы со структурой пирохлора $\mathrm{A}_{2} \mathrm{~B}_{2} \mathrm{O}_{7}$ (A редкоземельный ион, В - Ti, Ge, $\mathrm{Zr}, \mathrm{Sn}, \mathrm{Mo})$, в том числе станнаты, привлекают внимание благодаря многообразию свойств и потенциальному применению в качестве люминофоров [1-4]. Станнат иттрия $\mathrm{Y}_{2} \mathrm{Sn}_{2} \mathrm{O}_{7}$ используется в качестве матрицы для активации редкоземельными ионами. Исследуются люминесцентные свойства станната иттрия, активированного ионами $\mathrm{Eu}^{3+}$ и $\mathrm{Yb}^{3+}[5,6]$. Станнаты с подрешеткой редкоземельных ионов также активируют другими редкоземельными ионами [7]. В связи с этим представляет интерес исследование фононного спектра станнатов. Инфракрасные (ИК) спектры и спектры комбинационного рассеяния света (КРС) станната иттрия $\mathrm{Y}_{2} \mathrm{Sn}_{2} \mathrm{O}_{7}$ были измерены в работах $[6,8,9]$. Однако в низкочастотной области (до $200 \mathrm{~cm}^{-1}$ ) ИК спектр не был получен. Большая часть экспериментов была сделана на поликристаллических образцах, поэтому определение типов мод остается актуальным. В данной работе проведен $a b$ initio расчет фононного спектра $\mathrm{Y}_{2} \mathrm{Sn}_{2} \mathrm{O}_{7}$, определены частоты и типы ИК и КРС-мод. Упругие постоянные и твердость некоторых редкоземельных станнатов $\mathrm{R}_{2} \mathrm{Sn}_{2} \mathrm{O}_{7}(\mathrm{R}=\mathrm{La}$, $\mathrm{Nd}, \mathrm{Sm}, \mathrm{Gd}, \mathrm{Er}, \mathrm{Yb})$ недавно были измерены [10], однако для станната иттрия $\mathrm{Y}_{2} \mathrm{Sn}_{2} \mathrm{O}_{7}$ информация об упругих постоянных отсутствует. В данной работе были рассчитаны упругие постоянные $\mathrm{Y}_{2} \mathrm{Sn}_{2} \mathrm{O}_{7}$.

В работе также был проведен расчет расстояния „примесный ион-лиганд“ в примесных центрах $\mathrm{Y}_{2} \mathrm{Sn}_{2} \mathrm{O}_{7}: \mathrm{Eu}^{3+}, \mathrm{Y}_{2} \mathrm{Sn}_{2} \mathrm{O}_{7}: \mathrm{Yb}^{3+}$ и нескольких других.

Станнат иттрия $\mathrm{Y}_{2} \mathrm{Sn}_{2} \mathrm{O}_{7}$, как и редкоземельные станнаты $\mathrm{R}_{2} \mathrm{Sn}_{2} \mathrm{O}_{7} \quad(\mathrm{R}=\mathrm{La}-\mathrm{Lu})$, имеет структуру пирохлора, пространственная группа (№ 227). Катионы находятся в позициях: $\mathrm{Sn}-16 c(0,0,0), \mathrm{Y}(\mathrm{R})-$ $16 d(1 / 2,1 / 2,1 / 2)$. Кислород находится в двух симметрийно неэквивалентных позициях: О1 $-48 f(x, 1 / 8,1 / 8)$, $\mathrm{O} 2-8 b,(3 / 8,3 / 8,3 / 8)$.

\section{Методы расчета}

Расчеты проведены в рамках теории функционала плотности (DFT) с гибридными функционалами B3LYP и PBE0 [11], учитывающими вклад нелокального обмена в формализме Хартри-Фока. Сравнение функционалов различного уровня, сделанное в работе [12], показало, что PBE0 характеризуется малой ошибкой при воспроизведении электронной плотности и ряда других параметров относительно расчета методом связанных кластеров (CCSD). Функционалы B3LYP и PBE0 хорошо воспроизводят структуру и динамику решетки соединений с ионно-ковалентной связью $[13,14]$. Расчеты были проведены в программе CRYSTAL17 $[15,16]$, предназначенной для моделирования периодических структур в рамках МО ЛКАО подхода. Для кислорода был использован базис [17], содержащий в качестве диффузных и поляризационных $p$ - и $d$-орбитали. Для описания внутренних оболочек олова был использован релятивистский псевдопотенциал, внешние оболочки $4 s^{2} 4 p^{6} 4 d^{10} 5 s^{2} 5 p^{2}$, участвующие в химической связи, описывались посредством валентного базисного набора [18]. Для иттрия был использован псевдопотенциал [19], замещающий электроны первых трех слоев с валентным базисным набором TZVP-типа для описания внешних $4 s^{2} p^{6} d^{1} 5 s^{2}$-электронов. Базис кислорода, псевдопотенциалы олова и иттрия доступны на сайте программы CRYSTAL. Для описания внутренних оболочек редкоземельных ионов были использованы квазирелятивистские псевдопотен- 
циалы $\mathrm{ECP} n \mathrm{MWB}(\mathrm{ECP}$ — „effective core potential“; $n$ - количество внутренних электронов, замененных на псевдопотенциал; WB - „quasirelativistic“ $[20,21])$. Например, для европия $n=52$, для иттербия $n=59$. Для описания внешних оболочек $\left(5 s^{2} 5 p^{6}\right)$, участвующих в образовании химической связи, использовались валентные базисные наборы TZVP-типа с диффузными и поляризационными орбиталями [21-23]. Псевдопотенциалы и валентные базисные наборы („ЕCPnMWB-II“) доступны на сайте Stuttgart [24]. Из валентных базисных наборов были удалены гауссовы примитивы с показателем экспоненты менее, чем 0.1 a.u. ${ }^{-2}$, поскольку данные расчеты периодические.

Последовательность расчетов была следующей. При расчетах сначала проводилась оптимизация кристаллической структуры. Затем для оптимизированной кристаллической структуры, соответствующей минимуму энергии, выполнялся расчет фононного спектра или расчет упругих постоянных.

При решении системы одноэлектронных уравнений Кона-Шэма точность расчета самосогласованного поля была установлена $10^{-7}$ a.u. Точность расчета двухэлектронных интегралов - не менее $10^{-8}$ a.u. Интегрирование по зоне Бриллюэна проводилось по схеме Монкхорста-Пака с сеткой $k$-точек $10 \times 10 \times 10$.

В общем случае при релаксации кристаллической структуры учитывается, что в стационарной точке на поверхности потенциальной энергии силы, действующие на атомы, равны нулю. Задача решается численно, поэтому оптимизация считается завершенной, когда силы, действующие на атомы, становятся ниже порогового значения. В программе CRYSTAL для оценки величины сил на каждом шаге оптимизации рассчитывается среднеквадратичная величина градиента энергии и абсолютное значение его наибольшей компоненты (для них были заданы пороговые значения 0.00030 и 0.00045 a.u., что соответствует 0.015 и $0.023 \mathrm{eV} / \AA$ ). Кроме малости сил, в программе CRYSTAL используется еще один критерий - малость смещений ионов относительно предыдущего шага. То есть на каждом шаге оптимизации оцениваются смещения ионов относительно предыдущего шага. Оценка делается аналогичным образом по среднеквадратичному значению и абсолютному значению наибольшей компоненты (для них были заданы пороговые значения 0.0012 и 0.0018 a.u., что соответствует 0.00064 и $0.00095 \AA$ ). Таким образом, в программе CRYSTAL для завершения оптимизации необходимы выполнения условий малости сил, а также малости смещений ионов относительно предыдущего шага.

Частоты фононного спектра рассчитывались в Г-точке. B программе CRYSTAL фононный спектр рассчитывается в гармоническом приближении, при этом вычисляется динамическая матрица. Первые производные по смещениям ионов вычисляются аналитически, вторые численно [25]. Смещения ионов при расчете вторых производных задавались равными $0.003 \AA$ А. Интенсивность ИК и КРС активных мод в программе CRYSTAL [26] рассчитывается с использованием зарядов Борна [27]. При расчете упругих постоянных в программе CRYSTAL вычисляются вторые производные энергии ячейки по деформациям [26,28]:

$$
C_{i j}=\frac{1}{V}\left[\frac{\partial^{2} E}{\partial \varepsilon_{i} \partial \varepsilon_{j}}\right]_{0},
$$

при этом первые производные вычисляются аналитически, вторые численно: на кристаллическую ячейку накладывается деформация $\varepsilon_{i}$, и кристаллическая структура релаксирует при наложенной деформации.

\section{Обсуждение результатов}

Результаты расчета кристаллической структуры станната иттрия и ширины запрещенной щели (оценка „HOMO-LUMO“) в сравнении с экспериментальными данными приведены в табл. 1. Функционал B3LYP достаточно хорошо воспроизводит ширину запрещенной щели, однако завышает постоянную решетки. Расчет с функционалом РВЕ0 хорошо воспроизводит кристаллическую структуру. В целом, расчеты и с тем, и с другим функционалом дают удовлетворительное согласие с экспериментом.

Результаты расчета межионных расстояний и углов станната иттрия с функционалом РВE0 приведены в табл. 2, 3. В кристалле $\mathrm{Y}_{2} \mathrm{Sn}_{2} \mathrm{O}_{7}$ со структурой пирохлора катион $\mathrm{Y}^{3+}$ находится в искаженном восьмивершиннике из ионов кислорода. Из 8 ионов кислорода $6-$ это ионы О1 на расстоянии $\sim 2.49 \AA$ и два - ионы О2 на расстоянии $\sim 2.25 \AA$. Искажение кислородного окружения иттрия $\mathrm{Y}^{3+}$ в структуре пирохлора можно охарактеризовать отношением $\rho=\frac{\mathrm{R}-\mathrm{O} 2}{\mathrm{R}-\mathrm{O} 1}$.

Согласно экспериментальным данным [31], в ряду редкоземельных станнатов $\mathrm{R}_{2} \mathrm{Sn}_{2} \mathrm{O}_{7}(\mathrm{R}=\mathrm{La}-\mathrm{Lu})$ межионные расстояния уменьшаются: $\mathrm{R}-\mathrm{O} 1$ от 2.63 до 2.46, $\mathrm{R}-\mathrm{O} 2$ от 2.32 до 2.23, Sn-O1 от 2.09 до $2.05 \AA \AA$. Расчет предсказывает, что межионные расстояния в станнате иттрия наиболее близки к межионным расстояниям в станнатах эрбия $\mathrm{Er}_{2} \mathrm{Sn}_{2} \mathrm{O}_{7}$ и тулия $\mathrm{Tm}_{2} \mathrm{Sn}_{2} \mathrm{O}_{7}$. Это согласуется с тем, что близки ионные радиусы (по Шеннону) иттрия $(1.019 \AA)$, эрбия $(1.004 \AA)$ и тулия $(0.994 \AA)$.

Величина $\rho$, согласно экспериментальным данным [31], в ряду редкоземельных станнатов $\mathrm{R}_{2} \mathrm{Sn}_{2} \mathrm{O}_{7}$ $(\mathrm{R}=\mathrm{La}-\mathrm{Lu})$ возрастает от 0.881 до 0.907. Для станната иттрия $\mathrm{Y}_{2} \mathrm{Sn}_{2} \mathrm{O}_{7}$ расчет предсказывает $\rho$, равное 0.904 , практически такое же, как у станната эрбия. Согласно экспериментальным данным [31], в ряду редкоземельных станнатов $\mathrm{R}_{2} \mathrm{Sn}_{2} \mathrm{O}_{7}(\mathrm{R}=\mathrm{La}-\mathrm{Lu})$ угол $\mathrm{Sn}-\mathrm{O} 1-\mathrm{Sn}$ уменьшается от $131.61^{\circ}$ до $126.19^{\circ}$, угол $\mathrm{R}-\mathrm{O} 1-\mathrm{R}$ увеличивается от $92.05^{\circ}$ до $95.60^{\circ}$, угол $\mathrm{O} 1-\mathrm{Sn}-\mathrm{O} 1$ увеличивается от $96.47^{\circ}$ до $99.96^{\circ}$. Согласно расчетам, соответствующие углы в станнате иттрия близки к углам в станнатах эрбия $\mathrm{Er}_{2} \mathrm{Sn}_{2} \mathrm{O}_{7}$ и тулия $\mathrm{Tm}_{2} \mathrm{Sn}_{2} \mathrm{O}_{7}$. Можно предположить, что при активации станната иттрия эрбием $\mathrm{Er}^{3+}$ или $\mathrm{Tm}^{3+}$ искажение решетки будет минимальным. 
Таблица 1. Постоянная решетки, координата $x$ кислорода в позиции $48 f$, ширина запрещенной щели $\mathrm{Y}_{2} \mathrm{Sn}_{2} \mathrm{O}_{7}$

\begin{tabular}{l|c|c|c}
\hline \multicolumn{1}{c|}{ Параметр } & B3LYP & РBE0 & Эксперимент \\
\hline Постоянная решетки, $\AA$ & 10.507 & 10.411 & $10.373[29]$ \\
Координата $x$ (в долях постоянной решетки) & 0.3384 & 0.3384 & $0.338[29]$ \\
Ширина запрещенной щели, eV & 4.95 & 5.67 & $4.45[30]$
\end{tabular}

Таблица 2. Межионные расстояния $(\AA)$ в $\mathrm{Y}_{2} \mathrm{Sn}_{2} \mathrm{O}_{7}$. Расчет с функционалом PBE0

\begin{tabular}{c|c|c|c|c|c|c|c}
\hline \multicolumn{2}{c|}{$\mathrm{Sn}-\mathrm{O} 1$} & \multicolumn{2}{c|}{$\mathrm{Y}-\mathrm{O} 1$} & \multicolumn{2}{c}{$\mathrm{Y}-\mathrm{O} 2$} & \multicolumn{2}{c}{$\rho$} \\
\hline расчет & $\begin{array}{c}\text { экспери- } \\
\text { мент [32] }\end{array}$ & расчет & $\begin{array}{c}\text { экспери- } \\
\text { мент [32] }\end{array}$ & расчет & $\begin{array}{r}\text { экспи- } \\
\text { мент [32] }\end{array}$ & $\begin{array}{c}\text { расчет } \\
\text { экспери- } \\
\text { мент }\end{array}$ \\
\hline 2.0578 & $2.048 \pm 8$ & 2.4934 & $2.485 \pm 12$ & 2.2541 & $2.2457 \pm 10$ & 0.9040 & 0.9037
\end{tabular}

Таблица 3. Углы в структуре $\mathrm{Y}_{2} \mathrm{Sn}_{2} \mathrm{O}_{7}$ (в градусах, deg). Расчет РВЕ0. В скобках приведены данные эксперимента [32]

\begin{tabular}{c|c|c}
\hline $\mathrm{Sn}-\mathrm{O} 1-\mathrm{Sn}(\mathrm{deg})$ & $\mathrm{Y}-\mathrm{O} 1-\mathrm{Y}(\mathrm{deg})$ & $\mathrm{O} 1-\mathrm{Sn}-\mathrm{O} 1(\mathrm{deg})$ \\
\hline 126.85 & 95.14 & $99.55(99.4 \pm 1)$
\end{tabular}

Таблица 4. Заряды ионов и заряды на связях $\mathrm{Y}_{2} \mathrm{Sn}_{2} \mathrm{O}_{7}$ (по Малликену), $|e|$. Расчет РBE0. Результаты расчета c TZVP-базисом кислорода приведены в скобках

\begin{tabular}{c|c|c|c|c|c|c}
\hline $\mathrm{Y}$ & $\mathrm{Sn}$ & $\mathrm{O} 1$ & $\mathrm{O} 2$ & $\mathrm{Y}-\mathrm{O} 1$ & $\mathrm{Y}-\mathrm{O} 2$ & $\mathrm{Sn}-\mathrm{O} 1$ \\
\hline+2.000 & +1.881 & -1.089 & -1.227 & 0.022 & 0.046 & 0.153 \\
$(+1.836)$ & $(+1.919)$ & $(-1.021)$ & $(-1.181)$ & $(0.050)$ & $(0.060)$ & $(0.163)$
\end{tabular}

Результаты расчета кристаллической структуры хорошо согласуются с экспериментом [32] (табл. 2).

B программе CRYSTAL проводится анализ заселенностей по Малликену. Результаты анализа заселенностей по Малликену могут в существенной степени зависеть от базисного набора. Поэтому в работе был проведен тестовый расчет с TZVP-базисом кислорода [33]. Согласно расчетам (табл. 4), заряды ионов значительно отличаются от их зарядов в предположении ионной связи $(+3$ у иттрия, +4 у олова и +2 у кислорода). Однако зависимость результатов анализа заселенностей по Малликену от базисного набора (табл. 4) говорит в пользу того, что они носят скорее качественный характер.

Результаты расчета зонной структуры и плотности электронных состояний представлены на рис. 1. Расчет и с функционалом B3LYP, и с функционалом PBE0, предсказывает прямую $(\Gamma-\Gamma)$ щель. Согласно расчетам, потолок валентной зоны образован преимущественно состояниями кислорода, а дно зоны проводимости преимущественно состояниями иттрия.
Станнат иттрия $\mathrm{Y}_{2} \mathrm{Sn}_{2} \mathrm{O}_{7}$ имеет следующие фононные моды в Г-точке, характерные для структуры пирохлора: $\Gamma=A_{1 \mathrm{~g}}+E_{\mathrm{g}}+2 F_{1 \mathrm{~g}}+4 F_{2 \mathrm{~g}}+3 A_{2 \mathrm{u}}+3 E_{\mathrm{u}}+8 F_{1 \mathrm{u}}+4 F_{2 \mathrm{u}}$. Одна $F_{1 \mathrm{u}}$-мода трансляционная. Моды $4 F_{2 \mathrm{u}}, 3 E_{\mathrm{u}}, 3 A_{2 \mathrm{u}}$, $2 F_{1 \mathrm{~g}}$ - „молчащие“, не активные ни в ИК, ни в КРС. Моды $A_{1 \mathrm{~g}}+E_{\mathrm{g}}+4 F_{2 \mathrm{~g}}$ активны в КРС („рамановские“). Семь $F_{1 u}$-мод активны в ИК. Результаты расчета фононных мод станната иттрия в Г-точке приведены в табл. 5. Из $a b$ initio расчета были определены частоты и типы фононных мод. Из анализа векторов смещений, полученных при ab initio расчете, была определена степень участия ионов в каждой моде (столбец „Ионы-участники“ в табл. 5). Величины смещений ионов в фононных модах также представлены на рис. 2.

Расчет предсказывает, что максимальные смещения $\sim 0.04 \AA$ будут у иона иттрия в низкочастотной моде $F_{2 \mathrm{u}}\left(98 \mathrm{~cm}^{-1}\right)$, а также у ионов кислорода в $F_{2 \mathrm{u}}$-моде $\left(316 \mathrm{~cm}^{-1}\right)$ и в $F_{1 u^{-} \text {-моде }}\left(358 \mathrm{~cm}^{-1}\right)$. Если величина смещения составляет $0.02-0.04 \AA$, то смещение обозначено „S“, если величина смещения не превышает $0.01 \AA$, то смещение обозначено „ $W^{“}$. Если менее $0.005 \AA-$ ион не упоминается в столбце „Ионы-участники“ .

Согласно расчетам (рис. 2), ионы иттрия участвуют в модах с частотами до $\sim 300 \mathrm{~cm}^{-1}$, ионы олова в модах с частотами до $\sim 450 \mathrm{~cm}^{-1}$. Ионы кислорода проявляют участие во всем диапазоне частот, особенно находящиеся в позиции $48 f(\mathrm{O} 1)$. При этом можно выделить моды, в которых участвуют только ионы кислорода, либо моды, в которых они участвуют преимущественно. Во всех КРС-модах участвуют только ионы кислорода. В модах $E_{\mathrm{g}}\left(342 \mathrm{~cm}^{-1}\right)$ и $A_{\mathrm{g}}\left(497 \mathrm{~cm}^{-1}\right)$ участвуют только ионы кислорода, находящиеся в позиции $48 f(\mathrm{O} 1)$. Согласно расчетам (табл. 6), мода $A_{\mathrm{g}}\left(497 \mathrm{~cm}^{-1}\right)-$ наиболее интенсивная в КРС. Таким образом, изменение этой моды может говорить об изменении координаты $x$ кислорода, находящегося в позиции $48 f$. В максимальной по частоте ИК активной моде $F_{1 u}\left(633 \mathrm{~cm}^{-1}\right)$ преимущественно участвуют ионы кислорода, находящиеся в позиции $48 f$. В максимальной по частоте КРС- 

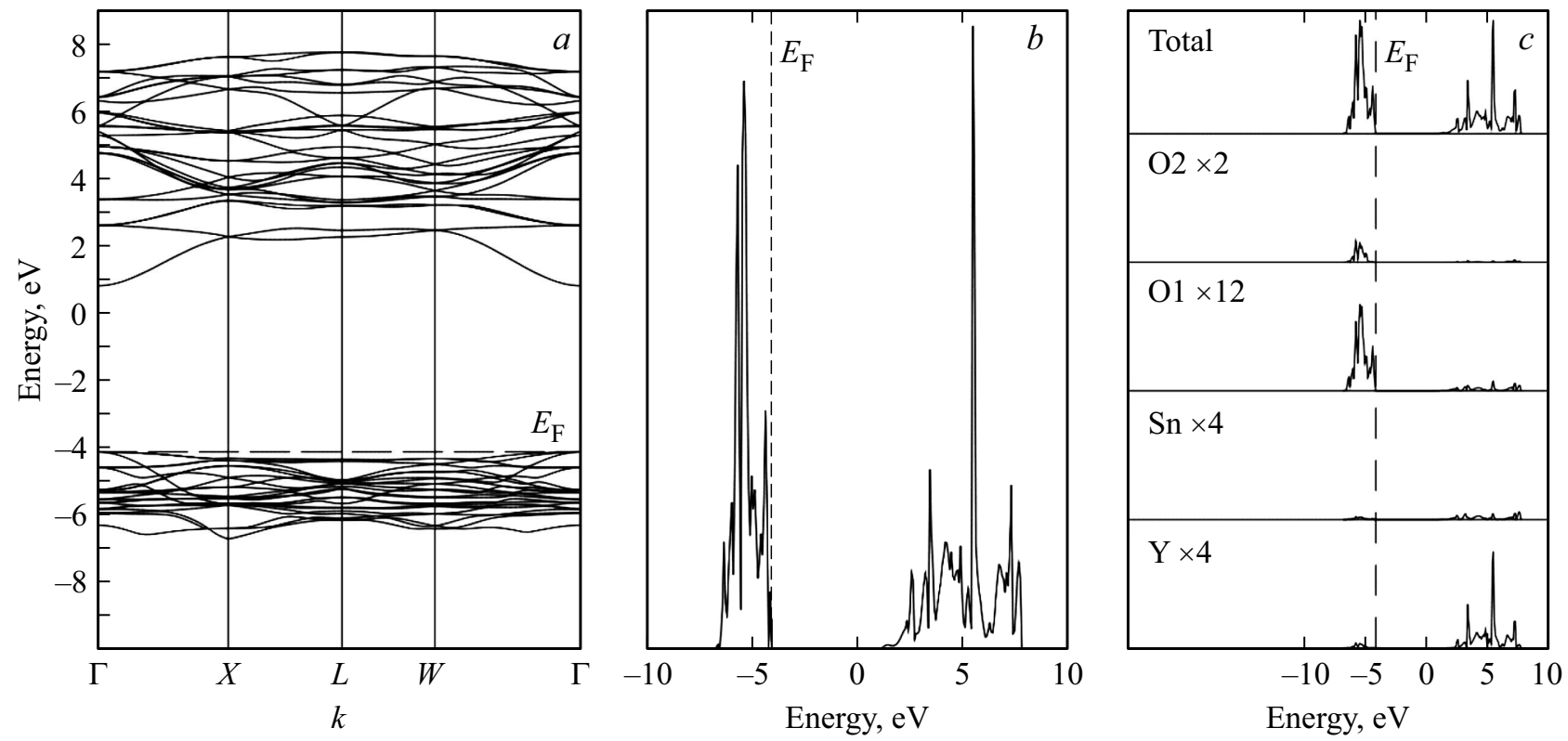

Рис. 1. Зонная структура $(a)$ и плотность электронных состояний (b и $c$ ) станната иттрия $\mathrm{Y}_{2} \mathrm{Sn}_{2} \mathrm{O}_{7}$. Расчет с функционалом B3LYP.
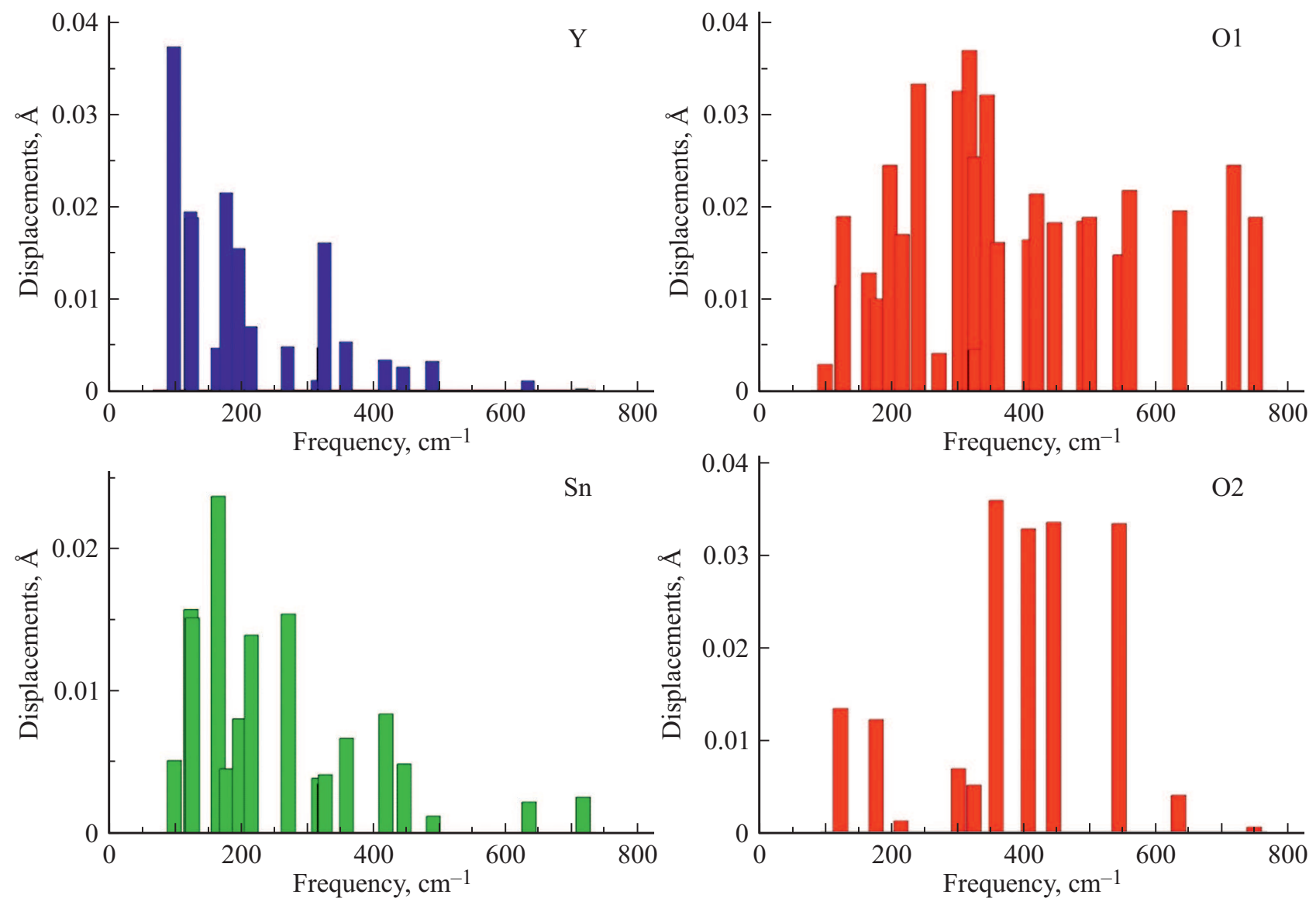

Рис. 2. Смещения ионов в фононных модах $\mathrm{Y}_{2} \mathrm{Sn}_{2} \mathrm{O}_{7}$. Расчет B3LYP. 
Таблица 5. Частоты $\left(\mathrm{cm}^{-1}\right)$ и типы фононных мод в Г-точке $\mathrm{Y}_{2} \mathrm{Sn}_{2} \mathrm{O}_{7}$. Обозначения в столбцах КРС и ИК: $A-$ активная мода, $I$ - неактивная. Расчет с функционалом B3LYP.

\begin{tabular}{|c|c|c|c|c|}
\hline Тип & ИК & КРС & $\begin{array}{c}\text { Частота, } \\
\text { расчет }\end{array}$ & Ионы-участники \\
\hline$F_{2 \mathrm{u}}$ & $I$ & $I$ & 98 & $\mathrm{Y}^{S}, \mathrm{Sn}^{W}$ \\
\hline$F_{1 \mathrm{u}}$ & $A$ & $I$ & 123 & $\mathrm{Y}^{S}, \mathrm{Sn}, \mathrm{O} 1, \mathrm{O} 2$ \\
\hline$E_{\mathrm{u}}$ & $I$ & $I$ & 126 & $\mathrm{Y}^{S}, \mathrm{Sn}, \mathrm{O} 1^{S}$ \\
\hline$F_{2 \mathrm{u}}$ & $I$ & $I$ & 164 & $\mathrm{Sn}^{S}, \mathrm{O} 1$ \\
\hline$F_{1 u}$ & $A$ & $I$ & 177 & $\mathrm{Y}^{S}, \mathrm{O} 1^{W}, \mathrm{O} 2$ \\
\hline$E_{u}$ & $I$ & $I$ & 196 & $\mathrm{Y}, \mathrm{Sn}^{W}, \mathrm{O} 1^{S}$ \\
\hline$F_{1 u}$ & $A$ & $I$ & 214 & $\mathrm{Y}^{W}, \mathrm{Sn}, \mathrm{O} 1$ \\
\hline$F_{1 \mathrm{~g}}$ & $I$ & $I$ & 239 & $\mathrm{O} 1^{S}$ \\
\hline$B_{\mathrm{u}}$ & $I$ & $I$ & 270 & $\mathrm{Sn}$ \\
\hline$F_{2 \mathrm{~g}}$ & $I$ & $A$ & 301 & $\mathrm{O} 1^{S}, \mathrm{O} 2^{W}$ \\
\hline$F_{2 \mathrm{u}}$ & $I$ & $I$ & 316 & $\mathrm{O} 1^{s}$ \\
\hline$F_{1 \mathrm{u}}$ & $A$ & $I$ & 324 & $\mathrm{O} 1^{S}, \mathrm{O} 2^{W}$ \\
\hline$B_{\mathrm{u}}$ & $I$ & $I$ & 326 & $\mathrm{Y}$ \\
\hline$E_{\mathrm{g}}$ & $I$ & $A$ & 342 & $\mathrm{O} 1^{S}$ \\
\hline$F_{1 \mathrm{u}}$ & $A$ & $I$ & 358 & $\mathrm{Y}^{W}, \mathrm{Sn}^{W}, \mathrm{O} 1, \mathrm{O} 2^{S}$ \\
\hline$F_{2 \mathrm{~g}}$ & $I$ & $A$ & 407 & $\mathrm{O} 1, \mathrm{O} 2^{S}$ \\
\hline$E_{\mathrm{u}}$ & $I$ & $I$ & 417 & $\mathrm{Sn}^{W}, \mathrm{O} 1^{S}$ \\
\hline$F_{1 \mathrm{u}}$ & $A$ & $I$ & 445 & $\mathrm{O} 1, \mathrm{O} 2^{S}$ \\
\hline$B_{\mathrm{u}}$ & $I$ & $I$ & 489 & $\mathrm{O} 1$ \\
\hline$A_{\mathrm{g}}$ & $I$ & $A$ & 497 & $\mathrm{O} 1^{S}$ \\
\hline$F_{2 \mathrm{~g}}$ & $I$ & $A$ & 543 & $\mathrm{O} 1, \mathrm{O} 2^{S}$ \\
\hline$F_{1 \mathrm{~g}}$ & $I$ & $I$ & 557 & $\mathrm{O} 1^{S}$ \\
\hline$F_{1 \mathrm{u}}$ & $A$ & $I$ & 633 & $\mathrm{O} 1^{S}$ \\
\hline$F_{2 \mathrm{u}}$ & $I$ & $I$ & 715 & $\mathrm{O} 1^{S}$ \\
\hline$F_{2 \mathrm{~g}}$ & $I$ & $A$ & 747 & $\mathrm{O}_{1}{ }^{S}$ \\
\hline
\end{tabular}

Примечание. Индексы в последнем столбце: $S$ - сильное, $W-$ слабое смещение иона в моде.

Таблица 6. Частоты $\left(\mathrm{cm}^{-1}\right)$ и типы КРС активных мод $\mathrm{Y}_{2} \mathrm{Sn}_{2} \mathrm{O}_{7}$. В столбце „Частота, расчет РВЕ0“ в скобках приведена интенсивность мод для поликристалла (в относительных единицах, rel. units). Расчет интенсивности КРС мод выполнен для возбуждающего излучения с длиной волны $514.4 \mathrm{~nm}$ и $T=298 \mathrm{~K}$

\begin{tabular}{c|l|c|c|l}
\hline Тип & $\begin{array}{l}\text { Частота, } \\
\text { расчет } \\
\text { РВЕ0 }\end{array}$ & $\begin{array}{c}\text { Частота, } \\
\text { расчет } \\
\text { В3LYР }\end{array}$ & $\begin{array}{c}\text { Частота, } \\
\text { экспери- } \\
\text { мент [6] }\end{array}$ & $\begin{array}{l}\text { Ионы- } \\
\text { участники }\end{array}$ \\
\hline$F_{2 \mathrm{~g}}$ & $311(904)$ & 301 & 311 & $\mathrm{O}^{S}, \mathrm{O} 2^{W}$ \\
$E_{\mathrm{g}}$ & $355(78)$ & 342 & 356 & $\mathrm{O}^{S}$ \\
$F_{2 \mathrm{~g}}$ & $414(347)$ & 407 & 412 & $\mathrm{O}^{S}, \mathrm{O}^{S}$ \\
$A_{1 \mathrm{~g}}$ & $512(1000)$ & 497 & 508 & $\mathrm{O}^{S}$ \\
$F_{2 \mathrm{~g}}$ & $545(82)$ & 543 & 563 & $\mathrm{O}^{S}, \mathrm{O}^{S}$ \\
$F_{2 \mathrm{~g}}$ & $761(64)$ & 747 & 628 & $\mathrm{O}^{S}$
\end{tabular}

моде $F_{2 \mathrm{~g}}\left(747 \mathrm{~cm}^{-1}\right)$ также преимущественно участвуют ионы кислорода, находящиеся в позиции $48 f$, однако интенсивность этой моды мала (табл. 6).

Расчеты (рис. 2) предсказывают смешивание колебаний структурных единиц. В ИК модах участвуют все ионы - иттрий, олово и кислород, но в разной

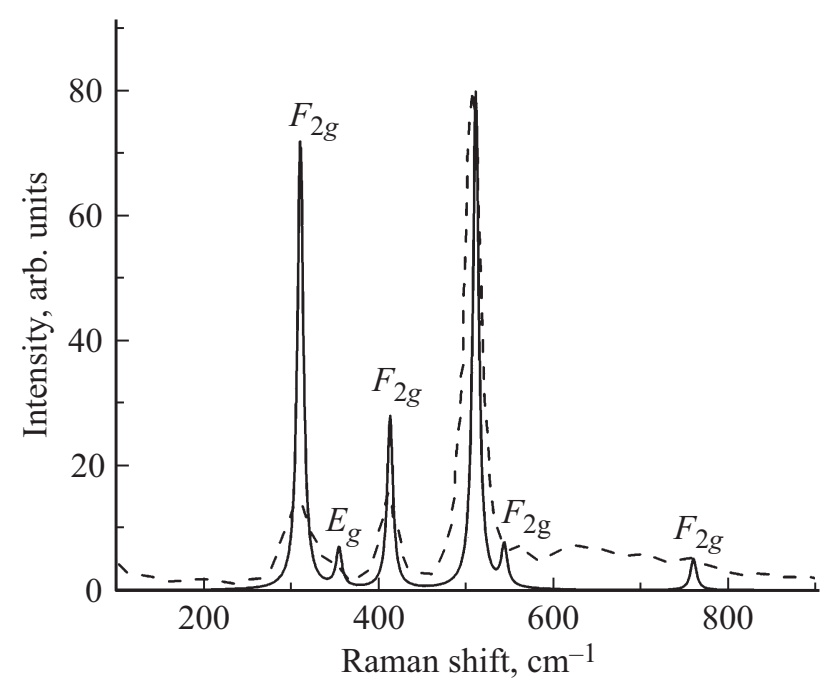

Рис. 3. КРС спектр $\mathrm{Y}_{2} \mathrm{Sn}_{2} \mathrm{O}_{7}$. Расчет (с функционалом РВЕ0) для возбуждающего излучения с длиной волны $514.4 \mathrm{~nm}$ и $T=298$ К. При моделировании КРС-спектра на основании рассчитанных частот и интенсивностей использовались функции Pseudo-Voigt c константой затухания (полушириной) $8 \mathrm{~cm}^{-1}$. Экспериментальный спектр (штриховая линия) [6] показан в таком масштабе, что высота максимального пика $\left(A_{1 g}\right)$ совпадает с высотой максимального пика в смоделированном спектре $\left(A_{1 g}\right)$. Расчет выполнен для поликристалла, что соответствует эксперименту.

степени. Сильное участие иттрия проявляется в двух низкочастотных $F_{1 \mathrm{u}}$-модах (123 и $177 \mathrm{~cm}^{-1}$ ) (табл. 7).

В табл. 6 и 7 приведены результаты расчета ИК и КРС-мод в сравнении с имеющимися экспериментальными данными. В работе [6] было указано только название спектрометра, использованного для измерения КРС-спектра. Длина волны возбуждающего излучения указана не была. Поэтому расчет интенсивности КРСмод был проведен для возбуждающего излучения с длиной волны $514.4 \mathrm{~nm}$ и $T=298 \mathrm{~K}$, что соответствует одному из режимов данного спектрометра. Результаты расчета КРС-мод (табл. 6) хорошо согласуются с экспериментом, за исключением высокочастотной моды $F_{2 \mathrm{~g}}$. Отметим, что, согласно и эксперименту [6], и расчету, эта мода обладает очень низкой интенсивностью (рис. 3). В табл. 7 приведены результаты расчета ИК мод в сравнении с экспериментом [6]. ИК спектр в работе [6] был снят в области частот более $200 \mathrm{~cm}^{-1}$, соответственно были определены шесть из семи ИК активных мод. Расчет дополнил его, предсказав моду $F_{1 u}$ с частотой $\sim 120 \mathrm{~cm}^{-1}$, с сильным участием иттрия. Результаты расчета ИК мод (табл. 7) достаточно хорошо согласуются с экспериментальными данными $[6,32,34,35]$.

В столбце „Вид колебания“ табл. 7 приведена интерпретация колебаний (валентные - „stretching“, деформационные - „bending“) на основе анализа векторов смещений, полученных при $a b$ initio расчете, и их 


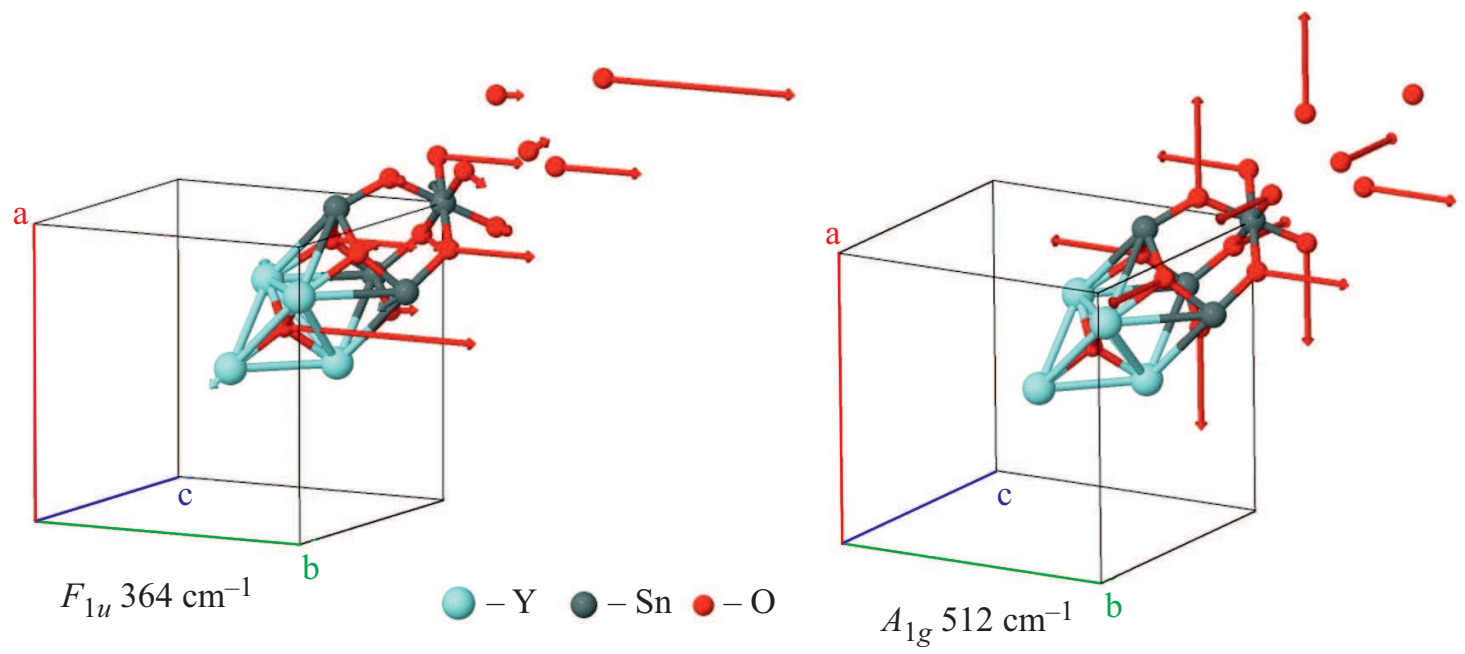

Рис. 4. Смещения атомов для наиболее интенсивных ИК и КРС-мод. Расчет РВЕ0.
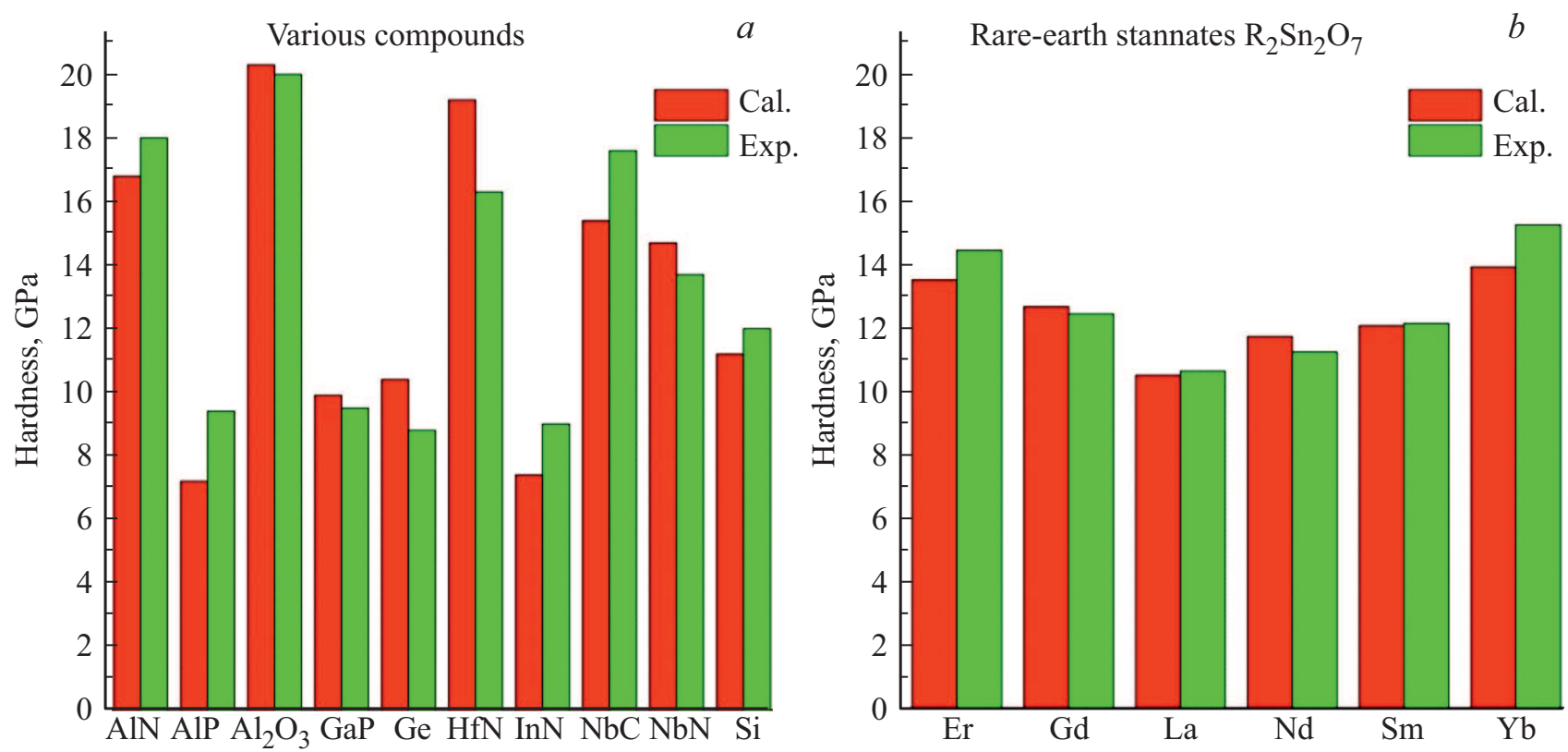

Рис. 5. Твердость по Виккерсу, рассчитанная по формуле (2) и измеренная непосредственно. $a$ - для ряда соединений, твердость которых по своей величине близка к твердости станнатов (график построен по данным работы [39]). $b$ - для редкоземельных станнатов: эксперимент из работы [10], расчет — по формуле (2) на основании измеренных в [10] упругих постоянных.

визуализации в программе Jmol [36]. Отметим, что в работе [29], где были обобщены экспериментальные данные для ряда станнатов, титанатов, цирконатов и других редкоземельных оксидов со структурой пирохлора, была проведена подобная интерпретация. В отличие от работы [29] $F_{1 u}$-моды с частотами 364 и $449 \mathrm{~cm}^{-1}$ в данной работе представляются как деформационные („bending").

На рис. 4 представлены наиболее интенсивная ИК и КРС-моды. В моде $F_{1 u}\left(364 \mathrm{~cm}^{-1}\right)$ участвуют преимущественно ионы кислорода. В моде $A_{1 g}\left(512 \mathrm{~cm}^{-1}\right)$ участвуют только ионы кислорода в позиции $48 f(\mathrm{O} 1)$.
В работе были рассчитаны упругие постоянные станната иттрия $\mathrm{Y}_{2} \mathrm{Sn}_{2} \mathrm{O}_{7}$. Это позволяет сделать оценку твердости кристалла. Для оценки твердости перхлоратов в работе [37] была успешно использована формула, выражающая твердость по Виккерсу $H_{V}$ через объемный модуль упругости и модуль сдвига. Эта эмпирическая формула была предложена в работе [38], исходя из того, что для поликристаллических образцов наблюдались корреляции между твердостью по Виккерсу и отношением модулей сдвига и объемного сжатия $G / B$. 
Таблица 7. ИК активные моды $\mathrm{Y}_{2} \mathrm{Sn}_{2} \mathrm{O}_{7}$. В скобках приведена интенсивность $(\mathrm{km} / \mathrm{mol})$

\begin{tabular}{|c|c|c|c|c|c|c|c|c|}
\hline \multirow[b]{2}{*}{ Тип } & \multicolumn{6}{|c|}{ Частота } & \multirow[b]{2}{*}{ Вид колебания } & \multirow{2}{*}{$\begin{array}{c}\text { Ионы- } \\
\text { участники }\end{array}$} \\
\hline & $\begin{array}{l}\text { расчет } \\
\text { PBE0 }\end{array}$ & $\begin{array}{l}\text { расчет } \\
\text { B3LYP }\end{array}$ & $\begin{array}{l}\text { экспери- } \\
\text { мент [6] }\end{array}$ & $\begin{array}{l}\text { экспери- } \\
\text { мент [32] }\end{array}$ & $\begin{array}{l}\text { экспери- } \\
\text { мент [34] }\end{array}$ & $\begin{array}{l}\text { экспери- } \\
\text { мент [35] }\end{array}$ & & \\
\hline$F_{1 u}$ & $120(533)$ & 123 & - & - & - & 125 & $\begin{array}{l}\mathrm{O} 1-\mathrm{Y}-\mathrm{O} 2 \text { bending } \\
\mathrm{O} 1-\mathrm{Sn}-\mathrm{O} 1 \text { bending }\end{array}$ & $\begin{array}{c}\mathrm{Y}^{S}, \mathrm{Sn}, \mathrm{O} 1 \\
\mathrm{O} 2\end{array}$ \\
\hline$F_{1 u}$ & $175(529)$ & 177 & 225 & - & - & 180 & $\begin{array}{c}\mathrm{Y}-\mathrm{Y} \text { stretching, } \\
\mathrm{O} 1-\mathrm{Sn}-\mathrm{O} 1 \text { bending }\end{array}$ & $\mathrm{Y}^{S}, \mathrm{O} 1^{W}, \mathrm{O} 2$ \\
\hline$F_{1 u}$ & $216(3301)$ & 214 & 240 & - & - & 230 & $\begin{array}{l}\mathrm{O} 1-\mathrm{Sn}-\mathrm{O} 1 \text { bending } \\
\mathrm{Y}-\mathrm{O} 1 \text { stretching }\end{array}$ & $\mathrm{Y}^{W}, \mathrm{Sn}, \mathrm{O} 1$ \\
\hline$F_{1 u}$ & $329(197)$ & 324 & 273 & - & - & - & $\begin{array}{l}\mathrm{O} 1-\mathrm{Sn}-\mathrm{O} 1 \text { bending } \\
\mathrm{Y}-\mathrm{O} 1 \text { stretching }\end{array}$ & $\mathrm{O} 1^{S}, \mathrm{O} 2^{W}$ \\
\hline$F_{1 u}$ & $364(8512)$ & 358 & 384 & 387 & - & 397 & $\begin{array}{l}\mathrm{Y}-\mathrm{O} 2-\mathrm{Y} \text { bending } \\
\mathrm{O} 1-\mathrm{Sn}-\mathrm{O} 1 \text { bending }\end{array}$ & $\begin{array}{l}\mathrm{Y}^{W}, \mathrm{Sn}^{W} \\
\mathrm{O} 1, \mathrm{O} 2^{S}\end{array}$ \\
\hline$F_{1 u}$ & $449(930)$ & 445 & 454 & 499 & 450 & 450 & $\begin{array}{c}\mathrm{Y}-\mathrm{O} 2-\mathrm{Y} \text { bending } \\
\mathrm{O} 1-\mathrm{Sn}-\mathrm{O} 1 \text { bending }\end{array}$ & $\mathrm{O} 1, \mathrm{O} 2^{S}$ \\
\hline$F_{1 u}$ & $647(2958)$ & 633 & 651 & 644 & 645 & 645 & $\begin{array}{c}\mathrm{Y}-\mathrm{O} 1 \mathrm{bending} \\
\mathrm{Sn}-\mathrm{O} 1 \text { stretching }\end{array}$ & $\mathrm{O} 1^{S}$ \\
\hline
\end{tabular}

Таблица 8. Упругие постоянные (GPa), модуль сдвига (GPa), модуль объемного сжатия (GPa), твердость (GPa) редкоземельных станнатов $\mathrm{R}_{2} \mathrm{Sn}_{2} \mathrm{O}_{7}$

\begin{tabular}{c|c|c|c|c|c|c|c|c}
\hline & $\begin{array}{c}C_{11} \\
\text { экспери- } \\
\text { мент [10] }\end{array}$ & $\begin{array}{c}C_{12} \\
\text { экспери- } \\
\text { мент [10] }\end{array}$ & $\begin{array}{c}C_{44} \\
\text { экспери- } \\
\text { мент [10] }\end{array}$ & $\begin{array}{c}B \\
\text { (по Хиллу) } \\
\text { расчет, } \\
\text { данная } \\
\text { работа }\end{array}$ & $\begin{array}{c}G \\
\text { (по Хиллу) } \\
\text { расчет, } \\
\text { данная } \\
\text { работа }\end{array}$ & $\begin{array}{c}H_{V} \\
\text { экспери- } \\
\text { мент [10] }\end{array}$ & $\begin{array}{c}H_{V} \\
\text { расчет, } \\
\text { данная } \\
\text { работа }\end{array}$ & $\begin{array}{c}\text { Различие, } \\
\%\end{array}$ \\
\hline $\mathrm{La}$ & 314.9 & 125.9 & 94.5 & 188.9 & 94.5 & $10.6 \pm 0.2$ & 10.48 & -1.13 \\
$\mathrm{Nd}$ & 337.3 & 130.1 & 103.6 & 199.2 & 103.6 & $11.2 \pm 0.4$ & 11.69 & 4.38 \\
$\mathrm{Sm}$ & 331.7 & 124.8 & 103.4 & 193.8 & 103.4 & $12.1 \pm 0.6$ & 12.03 & -0.58 \\
$\mathrm{Gd}$ & 333.9 & 122.2 & 105.9 & 192.8 & 105.9 & $12.4 \pm 0.2$ & 12.63 & 1.85 \\
$\mathrm{Er}$ & 340.1 & 120.2 & 109.9 & 193.5 & 109.9 & $14.4 \pm 0.3$ & 13.48 & -6.38 \\
$\mathrm{Yb}$ & 340.5 & 118.1 & 111.2 & 192.2 & 11.2 & $15.2 \pm 0.3$ & 13.87 & -8.75
\end{tabular}

Она имеет вид

$$
H_{V}=C\left(\frac{G}{B}\right)^{m} G^{n}
$$

В формуле (1) $H_{V}$ - твердость по Виккерсу, $G-$ модуль сдвига, $B$ - модуль объемного сжатия, $C$ коэффициент пропорциональности, $m$ и $n$ - эмпирические степенные коэффициенты. В работе [39] были определены параметры этой формулы, которые позволили описать твердость большого ряда соединений, более 40 , с различным типом химической связи, ионной и ковалентной:

$$
H_{V}=0.92\left(\frac{G}{B}\right)^{1.137} G^{0.708} .
$$

В формуле (2) используются модуль сдвига $G$ и модуль
Таблица 9. Упругие постоянные (GPa), модуль сдвига (GPa), модуль объемного сжатия (GPa), твердость (GPa) станната иттрия $\mathrm{Y}_{2} \mathrm{Sn}_{2} \mathrm{O}_{7}$

\begin{tabular}{c|c|c|c|c|c}
\hline$C_{11}$ & $C_{12}$ & $C_{44}$ & $\begin{array}{c}B \\
\text { (по Хиллу) }\end{array}$ & $\begin{array}{c}G \\
\text { (по Хиллу) }\end{array}$ & $H_{V}$ \\
\hline 336.9 & 111.5 & 97.3 & 186.6 & 103.2 & 12.5
\end{tabular}

объемного сжатия $B$, рассчитанные в приближении Хилла. В работе [10] были измерены упругие постоянные и твердость по Виккерсу ряда редкоземельных станнатов $\mathrm{R}_{2} \mathrm{Sn}_{2} \mathrm{O}_{7}(\mathrm{R}=\mathrm{La}, \mathrm{Nd}, \mathrm{Sm}, \mathrm{Gd}, \mathrm{Er}, \mathrm{Yb})$. Это дает возможность проверить применимость формулы (2) для редкоземельных станнатов. На основании измеренных в работе [10] упругих постоянных мы рассчитали модуль 
Таблица 10. Расстояния „,R-O1“ и „,R-O2“ в чистых кристаллах $\mathrm{Y}_{2} \mathrm{Sn}_{2} \mathrm{O}_{7}, \mathrm{Nd}_{2} \mathrm{Sn}_{2} \mathrm{O}_{7}, \mathrm{Nd}_{2} \mathrm{Sn}_{2} \mathrm{O}_{7}$ и соответствующие расстояния $(\AA)$ в примесных центрах $\mathrm{Y}_{2} \mathrm{Sn}_{2} \mathrm{O}_{7}: \mathrm{Eu}^{3+}, \mathrm{Y}_{2} \mathrm{Sn}_{2} \mathrm{O}_{7}: \mathrm{Yb}^{3+}, \mathrm{Nd}_{2} \mathrm{Sn}_{2} \mathrm{O}_{7}: \mathrm{Gd}^{3+}, \mathrm{Nd}_{2} \mathrm{Sn}_{2} \mathrm{O}_{7}: \mathrm{Tb}^{3+}, \mathrm{Lu}_{2} \mathrm{Sn}_{2} \mathrm{O}_{7}: \mathrm{Yb}^{3+} . \mathrm{Pac}^{2}$ с функционалом РВЕ0

\begin{tabular}{l|c|c|c|c}
\hline & \multicolumn{2}{|c|}{$\mathrm{R}-\mathrm{O} 1$} & \multicolumn{2}{c}{$\mathrm{O} 2$} \\
\cline { 2 - 5 } Кристалл & Расчет & $\begin{array}{c}\text { Изменение расстояния } \\
\text { в примесном центре } \\
\text { относительно чистой } \\
\text { решетки }\end{array}$ & Расчет & $\begin{array}{c}\text { Изменение расстояния } \\
\text { в примесном центре } \\
\text { относительно чистой } \\
\text { решетки }\end{array}$ \\
\hline $\mathrm{Y}_{2} \mathrm{Sn}_{2} \mathrm{O}_{7}$ & 2.4934 & - & 2.2541 & - \\
$\mathrm{Y}_{2} \mathrm{Sn}_{2} \mathrm{O}_{7}: \mathrm{Eu}^{3+}$ & 2.5182 & +0.0248 & 2.3058 & +0.0517 \\
$\mathrm{Y}_{2} \mathrm{Sn}_{2} \mathrm{O}_{7}: \mathrm{Yb}^{3+}$ & 2.4854 & -0.0080 & 2.2350 & -0.0191 \\
$\mathrm{Nd}_{2} \mathrm{Sn}_{2} \mathrm{O}_{7}$ & 2.5859 & - & 2.3040 & - \\
$\mathrm{Nd}_{2} \mathrm{Sn}_{2} \mathrm{O}_{7}: \mathrm{Gd}^{3+}$ & 2.5640 & -0.0190 & 2.2572 & -0.0468 \\
$\mathrm{Nd}_{2} \mathrm{Sn}_{2} \mathrm{O}_{7}: \mathrm{Tb}^{3+}$ & 2.5591 & -0.0268 & 2.2455 & -0.0585 \\
$\mathrm{Lu}_{2} \mathrm{Sn}_{2} \mathrm{O}_{7}$ & 2.4691 & - & 2.2418 & - \\
$\mathrm{Lu}_{2} \mathrm{Sn}_{2} \mathrm{O}_{7}: \mathrm{Yb}^{3+}$ & 2.4717 & +0.0026 & 2.2454 & +0.0036
\end{tabular}

сдвига и модуль объемного сжатия по Хиллу (табл. 8), при этом использовалась программа ELATE [40]. Это позволило рассчитать твердость по формуле (2) и сравнить ее с измеренной непосредственно (табл. 8). Значение твердости HV, рассчитанное по формуле (2), хорошо согласуется с измеренным непосредственно (табл. 8).

На рис. 5 представлены значения твердости $H_{V}$, измеренные непосредственно и рассчитанные по формуле (2) для ряда соединений, твердость которых по своей величине близка к твердости редкоземельных станнатов. Также на рис. 5 представлены данные для редкоземельных станнатов, приведенных в табл. 8. Как видно из рис. 5 , формула (2) для станнатов дает согласие с экспериментом даже лучшее, чем для ряда соединений из работы [39]. Поэтому использование формулы (2) для оценки твердости станната иттрия $\mathrm{Y}_{2} \mathrm{Sn}_{2} \mathrm{O}_{7}$ представляется вполне допустимым.

Упругие постоянные станната иттрия $\mathrm{Y}_{2} \mathrm{Sn}_{2} \mathrm{O}_{7}$, pacсчитанные с функционалом PBE0, а также твердость, рассчитанная по формуле (2), приведены в табл. 9. Зависимость модуля Юнга от направления в кристалле $\mathrm{Y}_{2} \mathrm{Sn}_{2} \mathrm{O}_{7}$ (рис. 6) показывает небольшую анизотропию упругих свойств.

Станнат иттрия и редкоземельные станнаты используются в качестве матриц для активации редкоземельными ионами. В работах [5-7] были исследованы примесные центры $\mathrm{Y}_{2} \mathrm{Sn}_{2} \mathrm{O}_{7}: \mathrm{Eu}^{3+}, \mathrm{Y}_{2} \mathrm{Sn}_{2} \mathrm{O}_{7}: \mathrm{Yb}^{3+}, \mathrm{Nd}_{2} \mathrm{Sn}_{2} \mathrm{O}_{7}: \mathrm{Gd}^{3+}$, $\mathrm{Nd}_{2} \mathrm{Sn}_{2} \mathrm{O}_{7}: \mathrm{Tb}^{3+}, \mathrm{Lu}_{2} \mathrm{Sn}_{2} \mathrm{O}_{7}: \mathrm{Yb}^{3+}$. Представляется актуальным в рамках $a b$ initio подхода определить, насколько расстояние „примесный ион-лиганд“ будет отличаться от соответствующего расстояния в чистой решетке. Эта информация может быть использована при дальнейшем расчете кристаллического поля на примесном ионе, например, в модели обменных зарядов [41], и исследовании его штарковской структуры.

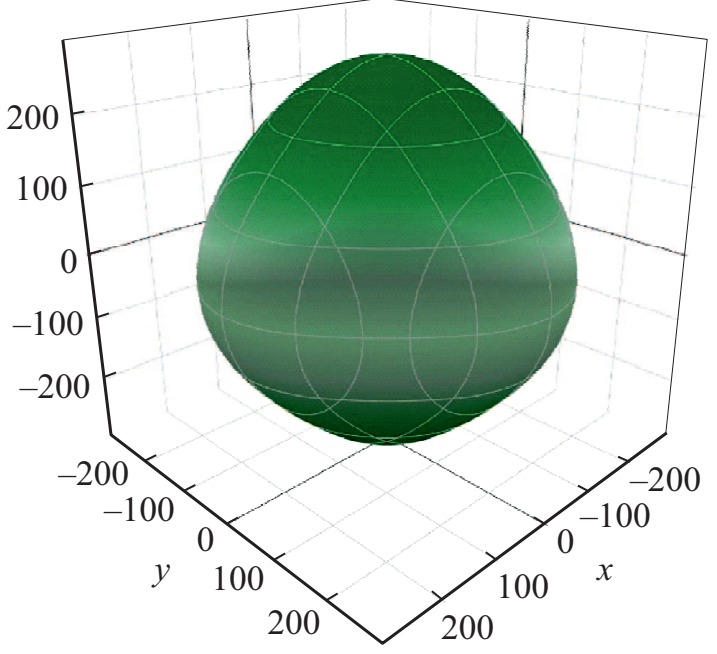

Рис. 6. Зависимость модуля Юнга $(\mathrm{GPa})$ от направления в кристалле $\mathrm{Y}_{2} \mathrm{Sn}_{2} \mathrm{O}_{7}$.

Для того чтобы смоделировать примесный центр, был проведен расчет, в котором один из четырех редкоземельных ионов в элементарной ячейке (или ионов иттрия) был заменен на примесный ион. Такой расчет позволил оценить локальные искажения решетки вблизи примесного иона. В табл. 10 приведены результаты расчета расстояний „примесный ион-лиганд“, а также соответствующие расстояния, рассчитанные для чистых кристаллов.

Как показывают расчеты (табл. 10), редкоземельные примесные центры в станнатах даже при изовалентном замещении могут вызывать существенные локальные искажения решетки. Согласно расчетам, расстояние „примесный ион-лиганд“ отличается от соответствующего расстояния в чистой решетке до $\sim 0.05 \AA$. 


\section{Заключение}

В результате работы из единого $a b$ initio расчета в рамках МО ЛКАО подхода были определены частоты и типы фундаментальных колебаний станната иттрия $\mathrm{Y}_{2} \mathrm{Sn}_{2} \mathrm{O}_{7}$, что позволило дополнить имеющиеся экспериментальные данные по ИК и КРС-спектрам. Из анализа векторов смещений, полученных из ab initio расчета, определена степень участия ионов в каждой моде. Определены моды с абсолютным или преимущественным участием кислорода в позиции $48 f$, характеризуемой координатой $x$. Показано, что в самой интенсивной КРС моде с частотой около $500 \mathrm{~cm}^{-1}$ участвуют только ионы кислорода, находящиеся в позиции $48 f$. Можно отметить, что поведение интенсивных мод $A_{1 g}\left(500 \mathrm{~cm}^{-1}\right)$ и $E_{g}\left(350 \mathrm{~cm}^{-1}\right)$ может нести информацию об изменении координаты $x$ кислорода в позиции $48 f$ под влиянием внешних воздействий на кристалл.

Рассчитаны упругие постоянные станната иттрия и твердость по Виккерсу; показано, что твердость $\mathrm{Y}_{2} \mathrm{Sn}_{2} \mathrm{O}_{7}$ близка к экспериментальным значениям твердости ряда редкоземельных станнатов $\mathrm{R}_{2} \mathrm{Sn}_{2} \mathrm{O}_{7}(\mathrm{R}=\mathrm{La}-\mathrm{Lu})$.

Показано, что редкоземельные примесные центры в станнатах, даже при изовалентном замещении, могут вызывать существенные локальные искажения решетки с изменением расстояния до лигандов $\sim 0.05 \AA$.

\section{Финансирование работы}

Работа выполнена при поддержке Министерства образования и науки РФ (проект № FEUZ-2020-0054). Работа выполнена в рамках государственного задания МИНОБРНАУКИ России (тема „Квант“, № AAAA-A18-118020190095-4). При проведении работ был использован суперкомпьютер „Уран“ ИММ УрО PAH.

\section{Конфликт интересов}

Авторы заявляют, что у них нет конфликта интересов.

\section{Список литературы}

[1] Hatnean M.C., Decorse C., Lees M.R., Petrenko O.A., Balakrishnan G. // Crystals. 2016. V. 6. N 7. P. 79. doi 10.3390/cryst6070079

[2] Cao R., Quan G., Shi Z., Chen T., Luo Z., Zheng G., Hu Z. // J. Phys. Chem. Solids. 2018. V. 118. P. 109. doi 10.1016/j.jpcs.2018.03.002

[3] Lian J., Chen J., Wang L.M., Ewing R.C., Farmer J.M., Boatner L.A., Helean K.B. // Phys. Rev. B. 2003. V. 68. N 13. doi 10.1103/PhysRevB.68.134107

[4] Srivastava A.M. // Opt. Mater. 2009. V. 31. N 6. P. 881. doi 10.1016/j.optmat.2008.10.021

[5] Jina D., Yu X., Yang H., Zhu H., Wang L., Zheng Y. // J. Alloys and Compounds. 2009. V. 474. P. 557. doi 10.1016/j.jallcom.2008.06.159
[6] Li K.W., Li H., Zhang H., Yu R., Wang H., Yan H. // Materials Research Bulletin. 2006. V. 41. P. 191. doi 10.1016/j.materresbull.2005.07.018

[7] Saleh A.A., Hamamera H.Z., Khanfar H.K., Qasrawi A.F., Yumusak G. // Materials Science in Semiconductor Processing. 2018. V. 88. P. 256. doi 10.1016/j.mssp.2018.08.017

[8] Pandey M., Nigam S., Sudarsan V., Kshirsagar R.J., Vatsa R.K. // AIP Conference Proceedings. 2014. V. 1591. P. 432. doi doi.org/10.1063/1.4872628

[9] Nayak C., Nigam S., Pandey M., Sudarsan V., Majumder C., Jha S.N., Bhatacharyya D., Vasta R.K., Kshirsagar R.J. // Chem. Phys. Lett. 2014. V. 597. P. 51. doi dx.doi.org/10.1016/j.cplett.2014.02.028

[10] Feng J., Xiao B., Qu Z.X., Zhou R., Pan W. // Appl. Phys. Lett. 2011. V. 99. doi 10.1063/1.3659482

[11] Perdew J.P., Ernzerhof M., Burke K. // J. Chem. Phys. 1996. V. 105. P. 9982. doi 10.1063/1.472933

[12] Medvedev M.G., Bushmarinov I.S., Sun J., Perdew J.P., Lyssenko K.A. // Science. 2017. V. 355. N 6320. P. 49. doi $10.1126 /$ science.aah5975

[13] Карпов В.В., Бандура А.В., Эварестов Р.А. // ФТТ. 2020. T. 62. № 6. C. 908. doi 10.21883/FTT.2020.06.49346.023

[14] Zhuravlev Y.N., Atuchin V.V. // Nanomaterials. 2020. V. 10. doi 10.3390/nano10112275

[15] Dovesi R., Saunders V.R., Roetti C., Orlando R., Zicovich-Wilson C.M., Pascale F., Civalleri B., Doll K., Harrison N.M., Bush I.J., D'Arco Ph., Llunel M., Causa M., Noel Y., Maschio L., Erba A., Rerat M., Casassa S. // CRYSTAL17 User's Manual. [Электронный ресурс] Режим доступа: http://www.crystal.unito.it/index.php

[16] [Электронный ресурс] Режим доступа: http://www.crystal.unito.it/index.php

[17] Cora F. // Mol. Phys. 2005. V. 103. N 18. P. 2483. doi 10.1080/00268970500179651

[18] Sophia G., Baranek P., Sarrazin C., Rerat M., Dovesi R. // Systematic Influence of Atomic Substitution on the Phase Diagram of ABO3 Ferroelectric Perovskites. 2014. [Электронный ресурс] Режим доступа: http://www.crystal.unito.it/Basis_Sets/tin.html

[19] Laun J., Oliveira D.V., Bredow T. // J. Comp. Chem. 2018. V. 39. N 19. P. 1285. doi $10.1002 /$ jcc.25195 (2018)

[20] Dolg M., Stoll H., Savin A., Preuss H. // Theor. Chim. Acta. 1989. V. 75. P. 173. doi $10.1007 / \mathrm{BF} 00528565$

[21] Dolg M., Stoll H., Preuss H. // Theor. Chim. Acta. 1993. V. 85. P. 441. doi 10.1007/BF01112983

[22] Yang J., Dolg M. // Theor. Chem. Acc. 2005. V. 113. P. 212. doi 10.1007/s00214-005-0629-0

[23] Weigand A., Cao X., Yang J., Dolg M. // Theor. Chem. Acc. 2009. V. 126. P. 117. doi 10.1007/s00214-009-0584-2

[24] Energy-consistent Pseudopotentials of the Stuttgart. [Электронный ресурс] Режим доступа: http://www.tc.uni-koeln.de/PP/clickpse.en.html

[25] Pascale F., Zicovich-Wilson C.M., Lopez Gejo F., Civalleri B., Orlando R., Dovesi R. // J. Comput. Chem. 2004. V. 25. P. 888. doi $10.1002 / \mathrm{jcc} .20019$

[26] Dovesi R., Orlando R., Erba A., Zicovich-Wilson C.M., Civalleri B., Casassa S., Maschio L., Ferrabone M., De La Pierre M., D'Arco P., Noel Y., Causa M., Rerat M., Kirtman B. // Int. J. Quantum Chem. 2014. V. 114. P. 1287. doi 10.1002/qua.24658

[27] Maschio L., Kirtman B., Orlando R., Rerat M. // J. Chem. Phys. 2012. V. 137. N 20. Art. no. 204113. doi $10.1063 / 1.4767438$ 
[28] Labeguerie P., Pascale F., Merawa M., Zicovich-Wilson C., Makhouki N., Dovesi R. // Eur. Phys. J. B. 2005. V. 43. P. 453. doi 10.1140/epjb/e2005-00078-6

[29] Subramanian M.A., Aravamudan G., Subba Rao G.V. // Prog. Solid St. Chem. 1983. V. 15. P. 55. doi 10.1016/0079-6786(83)90001-8

[30] Ambrosini A., Duarte A., Poeppelmeier K. P., Lane M., Kannewurf C.R., Mason T.O. // J. Solid State Chemistry. 2000. V. 153. P. 41. doi $10.1006 /$ jssc. 2000.8737

[31] Kennedy B.J., Hunter B.A., Howard C.J. // J. Solid State Chem. 1997. V. 130. P. 58. doi 10.1006/jssc.1997.7277

[32] Brisse F., Knop O. // Canadian J. Chemistry. 1968. V. 46. P. 859. doi $10.1139 / \mathrm{v} 68-148$

[33] Peintinger M.F., Oliveira D.V., Bredow T. // J. Computational Chemistry. 2013. V. 34. N 6. P. 451. doi $10.1002 /$ jcc. 23153

[34] Douma M., Chtoun El.H., Trujillano R., Rives V. // Proc. and Appl. of Ceramics. 2010. V. 4. N 4. P. 237. doi 10.2298/PAC1004237D

[35] McCaffrey J.F., McDevitt N.T., Phillippi C.M. // J. Optical Society of America. 1971. V. 61. Iss. 2. P. 209. doi 10.1364/JOSA.61.000209

[36] [Электронный pecypc] http://jmol.sourceforge.net/

[37] Корабельников Д.В., Журавлев Ю.Н. // ФТТ. 2016. T. 58. № 6. C. 1129; Korabelnikov D.V., Zhuravlev Yu.N. // Phys. Solid State. 2016. V. 58. N 6. P. 1166. doi 0.1134/S1063783416060251

[38] Chen X.Q., Niu H., Li D., Li Y. // Intermetallics. 2011. V. 19. P. 1275. doi 10.1016/j.intermet.2011.03.026

[39] Tian Y., Xu B., Zhao Z. // Int. J. Refractory Metals and Hard Materials. 2012. V. 33. P. 93. doi 10.1016/j.jirmhm.2012.02.021

[40] Электронный pecypc http://progs.coudert.name/elate

[41] Malkin B.Z. // Spectroscopy of Solids Containing Rare-earth Ions / Eds Kaplyanskii A.A., Macfarlane R.M. North-Holland, Amsterdam, 1987. P. 13 